Kai Wang · Lu Gan · Chia-Lam Kuo · Leroy Hood

\title{
A highly conserved apoptotic suppressor gene is located near the chicken T-cell receptor alpha chain constant region
}

\begin{abstract}
A cosmid clone containing the chicken T-cell receptor alpha chain constant region (TCRAC) was sequenced. The cosmid contains the TCRAC gene, six putative joining gene segments (TCRAJ), and surprisingly, a chicken homologue for the human apoptotic suppressor gene, defender against cell death (DADI). The DADl gene is 6.3 kilobases downstream of the TCRAC gene and has an inverted transcription orientation with respect to the TCRAC gene. The cDNA for the chicken DADl gene is 597 base pairs in length and encodes a highly conserved hydrophobic protein. The proximal location of $D A D 1$ to the TCRAC locus has also been confirmed in both humans and mouse. The location of the $D A D 1$ gene suggests that $D A D 1$ may play an important role in T-cell related apoptotic activities.
\end{abstract}

\section{Introduction}

Lymphocytes in the vertebrate immune system can be grouped into two major cell types; B and T cells based on their function and site of maturation. These cells execute the immune responses through their antigen-specific receptors. The antigen receptor for T cells, T-cell receptor (TCR), is a cell surface heterodimeric glycoprotein. The TCR is composed of either TCRA $(\alpha)$ and TCRB $(\beta)$ or TCRG $(\gamma)$

The nucleotide sequence data reported in this paper have been submitted to the GenBank nucleotide sequence database and have been assigned the accession numbers U83627, U83628, and U83833

K. Wang $(\bullet)^{1} \cdot$ L. Gan ${ }^{1} \cdot$ L. Hood

Department of Molecular Biotechnology, University of Washington, Box 357730, Seattle, WA 98195, USA

C.-L. Kuo

Institute of Biochemical Sciences, National Taiwan University, Box 23-106, Taipei, Taiwan, Republic of China

Present address:

1 Darwin Molecular Corp., 1631 220th St. SE., Bothell, WA 98021, USA and TCRD $(\delta)$ polypeptide chains. These chains are encoded by the TCRA, TCRB, TCRG, and TCRD gene families (reviewed in Hunkapiller and Hood 1989). Like the B-cell antigen receptor immunoglobulins, the TCR polypeptides are coded by multiple germline separated variable $(V)$, diversity $(D)$ (for TCRB and TCRD chain genes), joining $(J)$ gene segments, and constant region $(C)$ genes. During T-cell ontogeny, these gene segments are assembled at the DNA level through a site-specific recombination process.

The expression of the TCRA gene is largely regulated by the TCRA chain enhancer which is recognized by a set of complex trans-acting regulatory proteins (Diaz et al. 1994; Ho et al. 1990; Winoto and Baltimore 1989). The TCRA gene enhancer region is located approximately 4 kilobases (kb) downstream of the TCRA constant gene (Ho et al. 1990; Winoto and Baltimore 1989). Between humans and mouse, the 240 base pair (bp) enhancer region shares $86 \%$ of their nucleotide sequences (Koop et al. 1994). As well as the well-characterized TCRA chain enhancer, additional highly conserved DNA sequences called conserved sequence blocks (CSB) have been identified in the TCRAJ region through a sequence comparison between human and mouse TCRAJ-TCRAC region nucleotide sequences (Koop et al. 1994). Although nuclear proteins have been shown to recognize one of the conserved sequence blocks, the functions of these conserved regions remain to be determined (Koop et al. 1992, 1994; Hood et al. 1993).

Unique for T-lymphocyte development, the rearranged and expressed TCR repertoire is subsequently modified in thymus by both positive and negative selection processes (reviewed in Elliott 1993). Specifically, positive selection is the process whereby $\mathrm{T}$ cells are selected for maturation when the TCRs can recognize foreign antigens, and the negative selection eliminates self-reactive $T$ cells. In general, more than $98 \%$ of the lymphoid cells in thymus are driven to programmed cell death or apoptosis due to the negative selection process (Egerton et al. 1990). Several cellular encoded apoptotic effectors, such as Bcl-2, Bcl-x, and interleukin-1 $\beta$-converting enzyme (ICE) have been identified. However, little is known about their roles, if 
any, in the T-cell selection processes occurring in thymus (reviewed in Cory 1995).

Nucleotide sequences that are conserved through evolution include protein coding exons or important regulatory sequences. Therefore, comparing sequences from evolutionary diverged lines may assist in the identification process of genes and regulatory elements. Birds diverged from mammals approximately 300 to 350 million years ago. To study the evolution of the TCRAC gene and shed some light on the possible functions of previously identified conserved sequence blocks in human and mouse TCRAC region, a cosmid clone containing the chicken TCRAC gene region was isolated, sequenced, and analyzed. In this report, we describe the genomic organization of the chicken TCRAC gene and identification of a nearby human apoptotic suppressor gene homologue, defender against cell death (DADl).

\section{Materials and methods}

Nucleic acid and libraries

Chicken genomic DNA was prepared from liver using a standard protocol (Maniatis et al. 1989). A cosmid library with ten genomeequivalents was constructed from gnomic DNA in the pWE15A cosmid vector (Wang et al. 1995b). Total chicken thymus RNA was prepared from a freshly killed 3-month-old chicken using the guanidinium isothiocyanate extraction method (Chomczynski and Sacchi 1987). The poly(A) mRNA was selected through an oligo(dT)-cellulose column and cDNA was synthesized and cloned into $\lambda$-ZAP directional cloning vector (Stratagene, La Jolla, CA). A total of approximately $1 \times 10^{6}$ independent recombinant phage plaques were obtained and screened.

\section{Probes and hybridization}

Polymerase chain reactions [(PCR) (Saiki et al. 1988)] were used to obtain DNA probes. Oligonucleotide primers were synthesized from chicken TCRAC mRNA (Gobel et al. 1994) and chicken DADl germline sequences. The primer sequences were as follows:

\section{CHKCA1: CCTTCAGTCTACAGGCTGACC (for the chicken} TCRAC gene)

CHKCA1R: TCAGCATGTTCAGGTTCTCAT (for the chicken TCRAC gene)

CHKDAD1: AGTGGCACGTCGAGCCGTCTCA (for the chicken $D A D 1$ gene)

CHKDAD1R: GATGCAGGATGGTGTTGGCAAAG (for the chicken $D A D 1$ gene)

Approximately $1 \mathrm{ng}$ of chicken thymus cDNA was used in $50 \mu \mathrm{l}$ of PCR that consisted of $50 \mathrm{mM} \mathrm{NaCl}, 20 \mathrm{mM}$ Tris. $\mathrm{HCl}$ (pH 9.0), $2.5 \mathrm{mM}$ $\mathrm{M}_{\mathrm{g}} \mathrm{Cl}_{2}, 0.5 \mathrm{mM}$ dithiothreitol (DTT), $150 \mu \mathrm{M}$ of each nucleotide, $1 \mu \mathrm{M}$ of each gene-specific primer, and 2 units of Taq DNA polymerase. The reaction mixtures were subjected to 25 cycles of amplification. Each cycle consisted of $20 \mathrm{~s}$ at $94{ }^{\circ} \mathrm{C}, 45 \mathrm{~s}$ at $50{ }^{\circ} \mathrm{C}$, and $90 \mathrm{~s}$ at $72{ }^{\circ} \mathrm{C}$. The amplification products were analyzed by gel electrophoresis and the identity of the amplified products was determined by direct sequencing with $\gamma$-33P-ATP-labeled amplification primers.

For library screening, the purified fragment was labeled to a specific radioactivity greater than $1 \times 10^{8}$ c.p.m. $/ \mu \mathrm{g}$ of DNA by random priming (Feinberg and Vogelstein 1983). Membranes were hybridized overnight in $50 \%$ formamide $(\mathrm{V} / \mathrm{V}), 5 \times$ standard sodium citrate $[(\mathrm{SSC})(1 \times \mathrm{SSC}=0.15 \mathrm{M} \mathrm{NaCl} / 0.15 \mathrm{M}$ sodium citrate $)]$, $0.02 \mathrm{M}$ sodium phosphate (pH 6.7), $100 \mu \mathrm{g} / \mathrm{ml}$ denatured salmon sperm
DNA, $1 \%$ sodium dodecyl sulfate (SDS), $0.5 \%$ non-fat dry milk, and $10 \%$ dextran sulphate at $37^{\circ} \mathrm{C}$. Following hybridization, these membranes were washed twice in $2 \times$ SSC with $0.1 \%$ SDS at room temperature and twice in $0.5 \times \mathrm{SSC}$ with $0.1 \% \mathrm{SDS}$ at $65^{\circ} \mathrm{C}$. The filters were then blotted dry with Whatman $3 \mathrm{MM}$ filter paper and exposed to X-ray film overnight at $-70^{\circ} \mathrm{C}$ with intensifying screens.

\section{DNA sequencing}

The purified cosmid DNA was sonicated and DNA fragments 1.5 to $2 \mathrm{~kb}$ in size were purified from agarose gel as described (Wilson et al. 1992; Koop et al. 1994). The DNA fragments were cloned into Sma Idigested, dephosphorylated pUC18 vector. Sequencing template preparation and the subsequent sequencing reactions were carried out by PCR and cycle sequencing reactions, respectively (Wang et al. 1995a). Sequences were assembled with a test version of Sequencher DNA assembling program 3.0ß4 (Gene Codes Corp., Ann Arbor, MI) kindly provided by Gene Codes Corp prior to its release.

Sequence analysis

Sequence database searches and multi-sequence alignments were performed with BLAST, Inherit (ABI, Foster city, CA) and LaserGene sequence analysis program (DNA star, Madison, WI). The nucleotide and deduced amino acid sequences of the chicken $D A D 1$ gene were compared with those found in GenBank/EMBL (release 92).

\section{Results}

The chicken TCRAC gene containing the cosmid, clone 23.1, was sequenced by the shotgun sequencing approach as described (Wilson et al. 1992). The final assembled DNA sequence contains 31793 bp of genomic sequences. The sequence has about $45 \%$ of GC and does not contain any dior tri-nucleotide repeat with significant length (greater then five repeat units). The cosmid contains the entire chicken TCRAC gene, six putative TCRAJ gene segments, three copies of the chicken $C R l$ type repeat, and surprisingly, the chicken homologue of a gene which is thought to be a suppressor of apoptosis, DADl (Nakashima et al. 1993). Two copies of the CR1 repeat are located between the TCRAC gene and the DAD1 gene, and the other one resides between exon 1 and exon 2 of the $D A D 1$ gene (Fig. 1). The chicken CR1 repeat encodes a retroviral reverse transcriptase pseudogene. The transcriptional orientation of the CR1 repeats are indicated in Fig. 1.

Genomic organization of the chicken TCRAC and TCRAJ genes is similar to human and mouse

The TCRAC gene exons were identified by similarity comparisons with the chicken TCRA chain cDNA sequences (Gobel et al. 1994). The TCRAC gene in chicken, as in humans and mouse, is encoded by three translated exons, exon 1, 2, and 3, and an untranslated exon 4 . The exons are 264, 48, 107, and 474 bp in length and are separated by introns of 442,1070 , and $470 \mathrm{bp}$, respectively. The chicken TCRAC gene exons, like other known TCRAC genes, begin with the second codon position which insures 


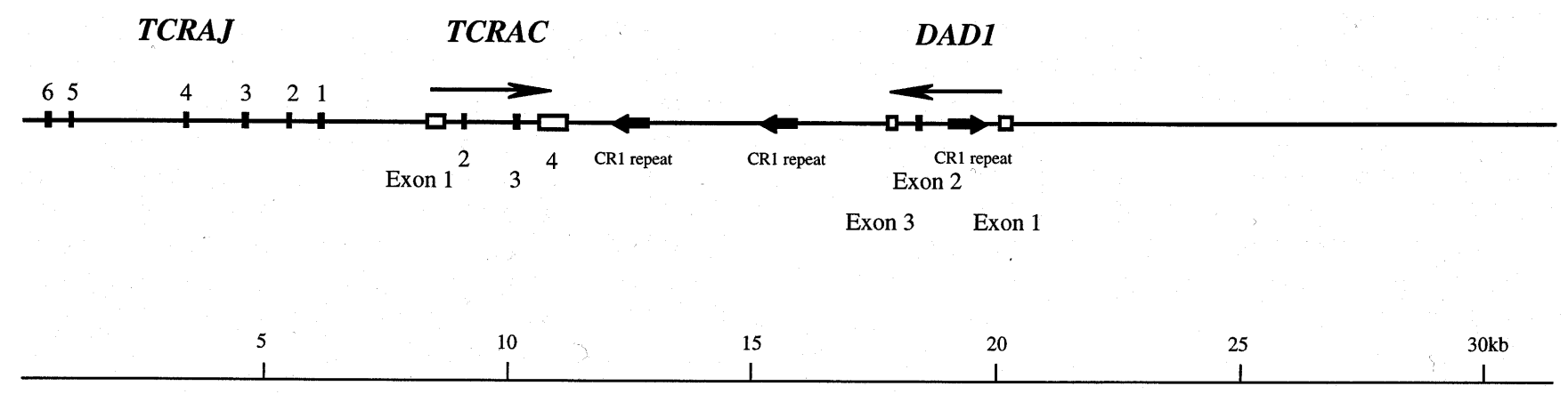

correct RNA splicing between constant gene exons and between the joining gene segment and the constant gene. Even though the chicken TCRAC gene share a similar intron/exon organization with human and mouse TCRAC genes, it shares little sequence similarity at either the nucleotide or the amino acid levels (Gobel et al. 1994). Amino acid residues that are important for the structural integrity of the TCRAC polypeptide are preserved in birds (Gobel et al. 1994).

To identify the chicken TCRAJ gene segments, the cosmid sequence was searched for $J$ gene segment core sequences, DNA recombination signals, and RNA splice sites as described (Koop et al. 1992, 1994). In addition, the cosmid sequence was compared with a file containing 122 previously identified human and mouse TCRAJ gene segment sequences (Koop et al. 1992, 1994). These searches resulted in the identification of six putative $J$ gene segments, TCRAJ1 to TCRAJ6 (Fig. 1). As in humans and mouse, the chicken TCRAJ gene segments are 50 to $63 \mathrm{bp}$ in length and are flanked by a 5' DNA recombination signal (GGTTTTTGT-11 to 12 bp spacer-CACTGTG) and a 3' RNA splice site (MAGGTRAG). The chicken $J$ gene segments also contain a centrally located conserved amino acid core sequence (Phe-Gly-X-Gly-Thr). All these putative TCRAJ gene segments are located upstream of the constant gene and have the same transcription orientation as the TCRA chain constant gene.
Fig. 1 Genomic organization of the chicken TCRAJ, TCRAC, and $D A D 1$ gene region. The TCRAJ gene segments and exons for TCRAC and $D A D 1$ genes are indicated. The CR1 repetitive sequences and its orientations are indicated with solid arrows. The arrows above the horizontal line indicate transcription orientations for the TCRAJ/ $T C R A C$ and $D A D 1$ genes

Alignment of the nucleotide sequences and deduced amino acid sequences of these TCRAJ gene segments are listed in Fig. 2. As expected, nucleotide sequence conservation at recombination signal and RNA splicing sites can be observed in chicken germline TCRAJ gene segments (Fig. 2A). Chicken TCRAJI and 2 are probably pseudogenes, since part of the conserved sequence motif does not present in the coding regions (Fig. 2B). However, the functionality of these $J$ gene segments can only be determined by expression studies such as cDNA library screening and sequencing.

Extensive nucleotide sequence conservation between the human and mouse TCRAC-TCRDC gene region has been

Fig. 2A, B Alignment of the chicken TCRAJ gene A nucleotide and $\mathbf{B}$ amino acid sequences. Consensus sequences are adapted from human and mouse TCRAJ sequences (Koop et al. 1994). Gaps have been introduced to maximize similarity between the sequences. Recombination signal (GTTTTTGTA---.--CACTGTG), J gene segment core sequence (TTTGGNNNNGGNAC), and the RNA splice signal (GTAAGT) are indicated

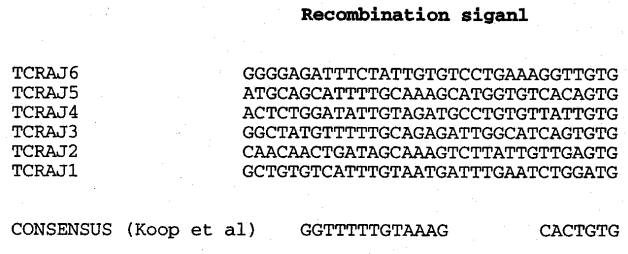

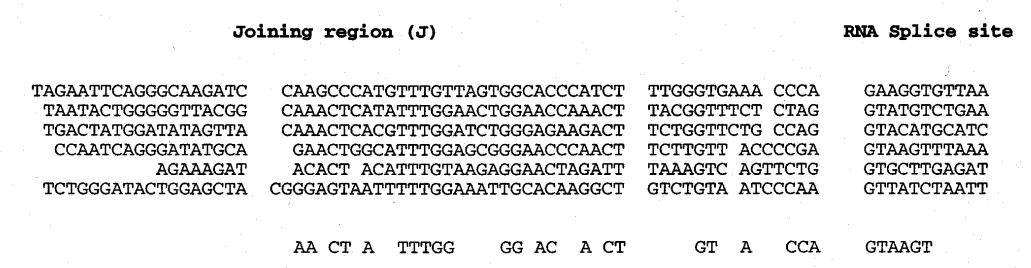

B

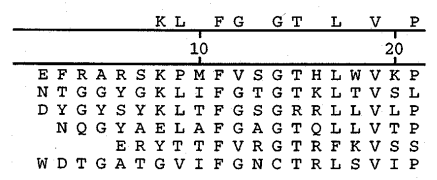

CONSENSUS 
Fig. 3 Germline nucleotide and deduced amino acid sequences of the chicken $D A D 1$ gene. The $D A D 1$ gene exons are identified by similarity comparison with the chicken DAD1 cDNA sequences. The exons are indicated and listed in capital letters. The deduced amino acids are listed above of the exons. Polyadenylation signals are in underlined boldface letters. The $\mathrm{CpG}$ islands and the RNA splice donor and acceptor sites are indicated in boldface characters. The chicken CR1 repeat sequence is underlined agaactgttactgtacctggaggagatacactgtctatactcccttgcagcagagagtagcctgggatgagtaaggtgga gaggaaacgctgtagttctcaatcatacaatcatagaatcattagagttggaaaaaactctaggatcacctactgcaac catcacccatcacataatgcttccctctggacataatataaagggacctacacaaaagcaacagttgcaggaatctgga agaagaaggatggcttttggatatcttcccagacatctcactgctgctgtgctggaccagagaggataccccaatgaata ttcccagagatgcacaaaaaacatacaattgacttgggagaaaactcccaggtctcatactgggcaatgcagataaagac tgggaaaccaacatgctcaggtctcagttttcttcaggaccgttgagacttcagcatgccaggcatcgtagaaggaaga cacataaaccccatactccatcctggcaaaaccaccccaccaatcccacctcctaccgccacgaactgatctcacacaa gggcacctctggagaccgagagacttctgcgcacgcgccagccggaagcacctttcagccaatccggcacgtacttatgc gcatgcgtattatctctagtaacacctcagttccgccctttgcgggcaggacaactgcgtttgcgcactatctctgacc cggaagtggactggccatgcgcgtgtgttgctcggctgtgtccoggaagcgcttgtcegctctgcttccggccacctcag Exon 1

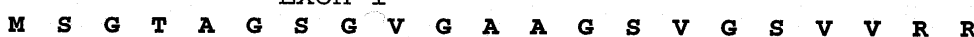

ctttcttctGGGGGGAGCATGTCGGGCACGGCGGGTTCGGGTGTGGGGGCTGCGGGCTCGGTAGGTTCTGTGGTGCGGCG $\begin{array}{llllllllllllllllllllllllll}\mathbf{F} & \mathbf{I} & \mathbf{A} & \mathbf{E} & \mathbf{Y} & \mathbf{G} & \mathbf{S} & \mathbf{G} & \mathbf{T} & \mathbf{S} & \mathbf{S} & \mathbf{R} & \mathbf{L} & \mathbf{K} & \mathbf{V} & \mathbf{L} & \mathbf{D} & \mathbf{A} & \mathbf{Y} & \mathbf{L} & \mathbf{L} & \mathbf{Y} & \mathbf{V} & \mathbf{M} & \mathbf{L} & \mathbf{T}\end{array}$ TTTCCTGGCGGAGTACGGCAGTGGCACGTCGAGCCGTCTCAAGGTGCTGGACGCATACCTGTTGTACGTGATGCTCACCG $\begin{array}{lllllllllllllllllllllllllll}\mathbf{G} & \mathbf{A} & \mathbf{L} & \mathbf{Q} & \mathbf{F} & \mathbf{G} & \mathbf{Y} & \mathbf{C} & \mathbf{L} & \mathbf{G} & \mathbf{V} & \mathbf{G} & \mathbf{T} & \mathbf{F} & \mathbf{P} & \mathbf{F} & \mathbf{N} & \mathbf{S} & \mathbf{F} & \mathbf{L} & \mathbf{S} & \mathbf{G} & \mathbf{F} & \mathbf{I} & \mathbf{S} & \mathbf{A} & \mathbf{V}\end{array}$ GGGCGCTCCAGTTTGGCTACTGCCTGGGTGTCGGCACCTTTCCCTTCAACTCCTTCCTCAGCGGCTTTATCTCCGCCGTC G $\mathbf{S} \mathbf{F} \mathbf{I} \mathbf{I}$

GGCAGCTTCATCCTCGGCGgtcagtgagaacCCCgcgcggttgcCCCCgacacccaggtcgtcccattaggatttctccg tggcgctgcttgaatctctcgcagtacccagggagctccccgcagtgcacgaagccccgtagctatcggagttccctcag tgacggtcacatagaaacacagaatggcttgaattggaagggacctcagagatcacctagttccagctcctctgatgtgg CR1 repeat

gctggttgctcatcaccagatcagattgcccatggccccatccagcctggccttgatcacctccggggtggggcatgcac cgctttttgggcagcttgttccagcaccttgccaccttctctgaaaaactttcccctgacatctcatctaagtctcccc tctcttagtttaaactattttcttcatctatcacttaactatgtaaaagttgatttccttcctatttttaatctccc tttaaatattggaaggccacaatgaggtctccccatagccctctcttctccaagctaaacaagcccagttcctccccagc tgtatt tataggagaggtgctccagccetctgagcatcctcatgoctctcctttgaaccctctccgaaaactcaacatct ttcttgtgttggggecccaaacctggatgcagtactctagatggggtctcacaaaggcagagcagaggggaacaatcacc tccctcaccctgatggccetcctctttgatgcagctcaggaaactgctggccttttgggatgcaaatgcacactgctgg ctcatgttaaggttttcctct tctgggacccccaaatccttctccacaggattgctctcaggragttctcccagactgtg tacatacctgggattgctctgactcaagtgcaacacttgtgcttggctttgttgaacgttattaggtttgtgtgggccc acctttgagtttgtcagtgtcccttggatgacatctcttacaccaactgcagacccaccttggccaaacccacagccc agggatcagtagcccccagtctgcccagcaactgggcagtgctggtgcttggcactccttgggtaattccagaccccttc tagaaaccagaaagttgccatagcaaagataaaaagcctaagaaagggtaaaacctgaaaactccaggatgttccctggt aaccttaaagcaaccagctctactcccagcatacccttctaggtctgtctgatggtgcccagatctgcaccccggcactg atggctggttctggcctgctggtcctaccccccagcaagatggtctgcagcagactgtgaacttatgtggttctgccctt gtgcaccacaaaacccagctcctcatgagccctatgtgtcccttcttctccagggtggtcagggaatcttccctttacc attttgtattgatgtctttcctagtgttcatcctatggtaaacccaaccctagcaataactgcttcaggatggtgcttc cctataatgcaccctttaaaaacaccatgtccctgtgcagcccttcccagcacagctaacagtgacctggacagggtgg

actgccttagctaggccatgcttcatgccatgaattccctttttgatctctgatgtatcattttctccctgctgtacag' Exon 2

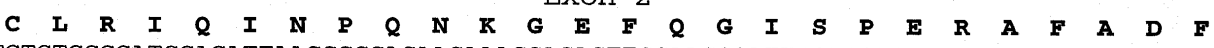
TTGTCTCCGGATCCAGATTAACCCCCAGAACAAAGGAGAGTTCCAAGGCATTTCACCAGAGAGGGCATTTGCTGATTTCC $\begin{array}{lllllllllllllllllllll}\mathbf{L} & \mathbf{F} & \mathbf{A} & \mathbf{N} & \mathbf{T} & \mathbf{I} & \mathbf{L} & \mathbf{H} & \mathbf{L} & \mathbf{V} & \mathbf{V} & \mathbf{I} & \mathbf{N} & \mathbf{F} & \mathbf{V} & \mathbf{G}\end{array}$

TCTTTGCCAACACCATCCTGCATCTCGTCGTTATCAATTTTGTTGGCTGAACTCTAGAAGAGTCTGAGgtactgatccat ggggccccagaaagggacgacactttctctacttttgtagatctgggagaaaactagaaacctcccaagctttctcatga gcaagtacagttaaaccattgttctttatgttgatagaggggtaagacttactgctttttgtttttatgaatttttattt ttttgctttattcaattaaaagtcagattaacttagtgtcacagaaatctaaccatattttactcttagagggatgaaaa atacctcaatttttctatgcagaaggaactttctgggctgagacttcaaggctctatccttgagggctgtcctgtttaga ggccatggaatggctggtatctaagtatgaagtttatcagcagatgagaggagatttaagaatagggagagactgataac agaatggggagagcatgttctgttaaagtttgtggcatatatttttttcccctttgcaaatgcattattcatgttatct Exon 3

ttctttcttccagGTCTTGAATTCTTCTAGAACTCTAATGTCAAATCCTGTTAGAGAGAAGTGTTGTTTTCCAGCAGTA CCTGCTGCTTTGGTGAGGAAGGGGAGAAGCAGAAGTCAAAGATTATTCCCACAGACTGTCCTATGGAGTGGTCTGTTGCC TCATTTCAGTTACTGTAATTCTTTCTTTAAATAAAGCCCTTCGTAATACTGCaatct t t tacctcttatgacaaagg gagagtttctcctgatgcttaagttcttgctcaggtgtttcctcctcctttctccttccctegggcttttttttcc observed (Koop et al. 1994). However, no significant sequence conservation on the orthologous regions has been identified through careful sequence comparison among the chicken, humans, and mouse.

\section{A highly conserved apoptotic suppressor gene, DADI is located downstream of the TCRAC gene}

Using BLAST and GRAIL search, a chicken homologue for the human apoptotic suppressor gene, DADl (Nakashima et al. 1993) was identified on the TCRAC gene containing cosmid sequence (Fig. 1). The chicken $D A D l$ gene is $6.3 \mathrm{~kb}$ downstream of the TCRAC gene and has an inverted transcription orientation with TCRAC and TCRA $J$ gene segments.

Chicken DAD1 cDNA is 697 bp in length and encodes a highly hydrophobic protein with 123 amino acid residues. Like its human orthologue, chicken $D A D 1$ is encoded by two translated exons, 1 and 2, and an untranslated exon 3 . The chicken $D A D 1$ gene exons are 250,149, and 198 bp in length and are separated by introns of 1659 and 506 bp, respectively. The germline nucleotide sequence and deduced amino acid sequence of the chicken $D A D 1$ gene is listed in Fig. 3. The chicken $D A D 1$ gene cDNA sequence contains a short 9 bp $5^{\prime}$ untranslated (UT) region and a 216 bp 3' UT region. Similar to the $D A D l$ gene in humans, expression of the $D A D 1$ gene in chicken can also be observed in all the tissues that have been studied thus far 


$\begin{array}{lll}\text { Human } & \text { DAD } & 1 \\ \text { Mouse } & \text { DAD } & 1 \\ \text { Rat } & \text { DAD } & 1 \\ \text { Chicken } & \text { DAD } & 1 \\ \text { Xenopus } & \text { DAD } 1 & \\ \text { C. elegans } & \text { DAD } & 1 \\ \text { Yeast OST 2 } & \text { DAD } 1 \text { like }\end{array}$

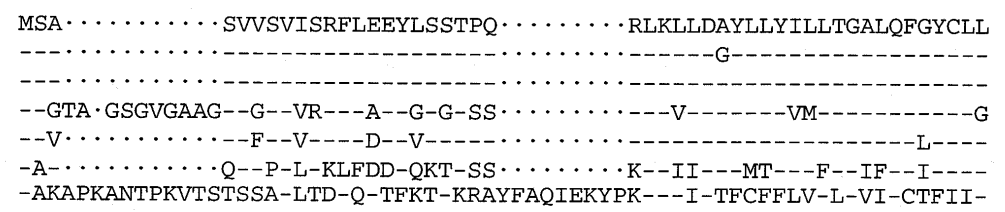

-AKAPKANTPKVTSTSSA LTD-Q-TEKT-KRAYFAOIEKYPK

$\begin{array}{lll}\text { Human } & \text { DAD } 1 \\ \text { Mouse } & \text { DAD } 1 \\ \text { Rat } & \text { DAD } 1 & \\ \text { Chicken } & \text { DAD } 1 \\ \text { Xenopus } & \text { DAD } 1 \\ \text { C. elegans } & \text { DAD } 1 & \\ \text { Yeast OST2 } & \text { DAD } 1 \text { like }\end{array}$

including spleen, thymus, brain, liver, and pancreas (data not shown).

Like other widely expressed genes, $\mathrm{CpG}$ islands can be identified in the $D A D 1$ promoter region (Fig. 3). Apart from the $\mathrm{CpG}$ islands, no other obvious regulatory sequences can be identified based on direct sequence analyses.

\section{The DADl gene is highly conserved through evolution}

Sequence alignment of chicken $D A D 1$ against other known $D A D 1$ genes and an yeast $D A D 1$ homologue, epsilonsubunit of the oligosaccharyltransferase (OST2) gene (Silberstein et al. 1995), is shown in Fig. 4. Over $60 \%$ of the DADI amino acid sequences are conserved from Caenorhabditis elegans to human. The chicken $D A D 1$ gene shares $80 \%$ of its amino acid sequence identity (Fig. 4) and $77 \%$ of coding region nucleotide sequence identity with the human $D A D 1$ gene (data not shown). In contrast, the nearby chicken TCRAC gene shares only $25 \%$ amino acid identity with its human counterpart (data not shown). The chicken $D A D 1$ gene has an insert of 10 codons in its $5^{\prime}$ region, unlike other $D A D 1$ genes (Fig. 4). The yeast OST2 gene shares approximately $40 \%$ and $30 \%$ of its peptide sequence with human and chicken $D A D 1$ sequences, respectively (Fig. 4).

\section{Discussion}

Comparing the coding region sequences of the TCRAJ and TCRAC gene between chicken and humans revealed only limited overall nucleotide sequence conservation. Regulatory sequences required for $T C R$ gene assembly are conserved between chicken and human. These include recombination signals as well as RNA splice donor and acceptor sites. Other sequences such as the core sequence motif for $J$ gene segments and key amino acid residues which are required for maintaining structural integrity of the TCRA chain polypeptide are also preserved through evolution. In contrast to the TCRA chain gene, the apoptotic suppressor gene, $D A D 1$ revealed extreme sequence conservation at both the nucleotide and the amino acid levels (Fig. 4).
Fig. 4 Amino acid sequence alignment for $D A D 1$ genes and its yeast homologue. Dashes (-) are used to indicate sequence identity with the human DADl sequence. Dots $(\cdot)$ are used to indicate gaps that were introduced by alignment program to maximize sequence similarity. The GenBank accession numbers for the sequences used in the alignment are: human D15057 (Nakashima et al. 1993), mouse U83628 (K. Wang and co-workers, in preparation), rat D15058 (Nakashima et al. 1993), chicken U83627, Xenopus B54437 (Nakashima et al. 1993), C. elegans X89080 (Sugimoto et al. 1996), and yeast OST2 U32307 (Silberstein et al. 1995)

Analyzing the genomic organization of the TCRAC gene in bird revealed a striking conservation in intron and exon structure

Even with 300 to 350 million years' separation and low overall nucleotide sequence conservation, the chicken TCRAC gene, like its human counterpart, is still encoded by four separated exons, three coding (exon 1 to 3 ), and one noncoding (exon 4). The protein translation frame in the chicken TCRAC gene, as in humans and mouse, begins with the second codon position which insures correct RNA splicing between constant gene exons and between TCRAJ and constant genes. The conservation of intron/ exon structure and protein translation reading frame clearly indicates that the TCRAC genes in birds and mammals are evolved from a common origin.

Sequence analyses of the TCRAC gene region in chicken failed to recognize the previously identified TCRA chain enhancer and conserved sequence blocks (CSB). This may imply that 1) the enhancer region and CSBs may not have any regulatory roles in the expression of the TCRA chain gene, 2) the avian TCRA chain gene may use different set of regulatory elements, 3) we may not be comparing the orthologous region, and more likely, 4) the core sequences for TCRA chain enhancer and other putative regulatory regions such as CSBs are much smaller then previously suggested and are not revealed by direct sequence comparison. Thus, biological studies such as transfection and deletional analysis may be required to locate the avian $T C R A$ locus regulatory regions. 
Extensive sequence conservation through evolution suggests that the function of DADI is fundamental for the cell

Unlike TCRAC, the DADI gene is small and highly conserved. Between humans and rat, the DADl gene is $99 \%$ identical at the protein level. Other apoptotic proteins such as $\mathrm{Bcl}-2$ do not show such extreme sequence conservation. Recently, the $D A D 1$ orthologue has been identified in $C$. elegans and even in plants (Sugimoto et al. 1996). The $D A D 1$ polypeptide is highly hydrophobic, which suggests that $D A D 1$, like the $\mathrm{Bcl}-2$ gene product, may be associated with sub-cellular compartmental membranes. As in humans, chicken $D A D 1$ gene expression was detected in all the tissues tested. Ubiquitous expression and extreme sequence conservation suggests that the $D A D l$ gene may play an essential and fundamental role in the survival of eukaryotic cells.

Yeast epsilon-subunit of the oligosaccharyltransferase has been cloned recently (Silberstein et al. 1995). Surprisingly, the yeast epsilon-subunit of the oligosaccharyltransferase protein shared $40 \%$ of its peptide sequence identity with the human $D A D 1$ protein. This finding suggests that $D A D 1$-mediated apoptotic suppression may be involved in the glycosylation and mannose transport pathways in the cell.

The human DADl gene is also located downstream of the TCRA chain constant gene

The human $D A D 1$ gene has been mapped to the chromosome 14q11-12 region by YAC and fluorescent in situ hybridization (Ylug et al. 1995). Our sequencing data in chicken raises the possibility that the $D A D 1$ gene may also be located near the TCRA chain constant gene in human. Genomic cloning and long range PCR confirm this suggestion (K. Wang and co-workers, manuscript in preparation). Since the coding region of the $D A D l$ gene in humans and chicken shares more than $80 \%$ of their sequence identity, it will be interesting to see whether this sequence conservation extends to introns and the $5^{\prime}$ and $3^{\prime}$ flanking regions of this gene.

The location of the DAD1 gene implies that it may play some role in the $T$-cell selection process

Negative selection processes play a key role in the maturation of cellular immunity. A cluster of cytotoxic T-cellspecific serine proteases, granzymes, have been mapped to human chromosome 14q11 near the TCRA locus (Harper et al. 1988; Hanson et al. 1990; Caughey et al. 1993). It is not unusual in cells that functionally related genes are closely linked in the genome. From studies of the serine protease cluster, it has been proposed that this chromosomal region, from TCRA to the granzyme cluster, is coordinately regulated during early stages of T-cell development (reviewed in Cory 1995). The identification of an apoptotic suppressor $D A D 1$ gene downstream of the TCRAC gene suggests that
$D A D 1$ may also play role in the early T-cell development processes.

The isolation of TCRA chain genes from evolutionary diverged species usually depends on cross-species hybridization or utilizes degenerate primers in PCR. These methods in general are difficult, unspecific, and time-consuming due to the low sequence conservation of the TCRA chain gene. The nearby $D A D 1$ gene, in contrast, is highly conserved through evolution. Thus, it may be possible to obtain the TCRAC gene from other species by taking advantage of the $D A D 1$ gene's extreme sequence conservation and proximal location to the TCRA locus.

Acknowledgments We are grateful to the sequencing group in the Department of Molecular Biotechnology at the University of Washington for their suggestions on DNA sequencing. We thank Gene Codes Corp. for providing us with a copy of the Sequencher DNA sequence assembling program prior to its release. We also thank Dr. Richard Gelinas and Mr. Patrick Moss for critical reading of the manuscript. Part of this work was supported by a DOE genome award to $\mathrm{L}$. H.

\section{References}

Caughey, G. H., Schaumberg, T. H., Zerweck, E. H., Butterfield, J. H., Hanson, R. D., Silverman, G. A., and Ley, T. J. The human mast cell chymase gene (CMA1):mapping to the cathepsin G/granzyme gene cluster and lineage-restricted expression. Genomics 15: 614620, 1993

Chomczynski, P. and Sacchi N. Single-step method of RNA isolation by acid guanidinium thiocyanate phenol chloroform extraction. Anal Biochem 162: 156-159, 1987

Cory, S. Regulation of lymphocyte survival by the Bcl-2 gene family. Annu Rev Immunol 13: 513-543, 1995

Davis, M. M. T cell receptor gene diversity and selection. Annu Rev Biochem 59: 475-496, 1990

Diaz, P., Cado, D., and Winoto, A. A locus control region in the T-cell receptor alpha/delta locus. Immunity 1: 207-217, 1994

Egerton, M., Scollay, R., and Shortman, K. Kinetics of mature T cell development in the thymus. Proc Natl Acad Sci USA 87: 25892592, 1990

Elliott, J. I. Thymic selection reinterpreted. Immunol Rev 135: 227242, 1993

Feinberg, A. P. and Vogelstein, B. A technique for radiolabelling DNA restriction fragments to high specific activity. Anal Biochem 132: 6-13, 1983

Gobel, T. W. F., Chen, C. L., Lahti, J., Kubota, T., Kuo, C. L., Aebersold, R., Hood, L., and Cooper, M. D. Identification of Tcell receptor alpha (TCR $\alpha)$ chain genes in the chicken. Proc Natl Acad SCI USA 91: 1094-1098, 1994

Hanson, R. D., Hohn, P. A., Popeseu, N. C., and Ley, T. J. A cluster of hematopoietic serine protease genes is found on the same chromosomal band as the human $\alpha / \delta$ T-cell receptor locus. Proc Natl Acad Sci USA 87: 960-963, 1990

Harper, K., Mattei, M., Simon, D., Suzan, M., Guenet, J., Haddad, P., Sasportes, M., and Golstein, P. Proximity of the CTLA-1 serine esterase and Tcr $\alpha$ loci in mouse and man. Immunogenetics 28: 439-444, 1988

Ho, I. C., Bhat, N. K., Gottschalk, L. R., Lindsten, T., Thompson, C. B., Papas, T. S., and Leiden, J. M. Sequence-specific binding of human Ets-1 to the T cell receptor $\alpha$ gene enhancer. Science 250: 814-818, 1990

Hood, L., Koop, B. F., Rowen, L., and Wang, K. Human and mouse Tcell receptor loci: the importance of comparative large-scale DNA sequence analyses. Cold Spring Harb Symp Quant Biol 58: 339348, 1993 
Hunkapiller, T. and Hood, L. Diversity of the immunoglobin gene superfamily. Adv Immunol 44: 1-63, 1989

Koop, B. F., Wilson, R. K., Wang, K., Vernooij, B., Zaller, D., Kuo, C. L., Seto, D., Toda, M., and Hood, L. Organization, structure, and function of $95 \mathrm{~kb}$ of DNA spanning the murine T-cell receptor $\mathrm{C} \alpha /$ C $\delta$ region. Genomics 13: 1209-1230, 1992

Koop, B. F., Rowen, L., Wang, K., Kuo, C. L., Seto, D., Lenstra, J. A., Howard, S., Shan, W., Deshpande, P., and Hood, L. The human Tcell receptor TCRAC/TCRDC $(\mathrm{C} \alpha / \mathrm{C} \delta)$ region: organization, sequence, and evolution of $97.6 \mathrm{~kb}$ of DNA. Genomics 19: 478-493, 1994

Maniatis, T., Fritsch, E. F., and Sambrook, J. Molecular Cloning: A Laboratory Manual, (2nd edn), Cold Spring Harbor Laboratory, Cold Spring Harbor, 1989

Nakashima, T., Sekiguchi, T., Kuraoka, A., Fufushima, K., Shibata, Y., Komiyama, S., and Nishimoto, T. Molecular cloning of a human cDNA encoding a novel protein, DAD1, whose defect causes apoptotic cell death in hamster BHK21 cells. Mol Cell Biol 13: 6367-6374, 1993

Saiki, R. K., Gelfand, D. H., Stoffel, S., Scharf, S. J., Higuchi, R., Horn, G. T., Mullis, K. B., and Erlich, H. A. Primer-directed enzymatic amplification of DNA with a thermostable DNA polymerase. Science 239: 487-491, 1988
Silberstein, S., Collins, P.G., Kelleher, D.J., and Gilmore, R. The essential OST2 gene encodes the $16-\mathrm{kD}$ subunit of the yeast oligosaccharyltransferase, a highly conserved protein expressed in diverse eukaryotic organisms. J Cell Biol 131: 371-383, 1995

Sugimoto, A., Hozak, R. R., Nakashima, T., Nishimoto, T., Rothman, J. H. DAD1, an endogenous programmed cell death suppressor in Caenorhabditis elegans and vertebrates. EMBO J 14: 4434-4441, 1996

Wang, K., Gan, L., Boysen, C., and Hood, L. A microtiter plate-based high-throughput DNA purification method. Anal Biochem 226: 8590, 1995a

Wang, K., Gan, L., Lee, I., and Hood, L. Isolation and characterization of the chicken trypsinogen gene family. Biochem J 307: 471-479, $1995 b$

Wilson, R. K., Koop, B. F., Chen, C., Halloran, N., Sciammis, R., and Hood, L. Nucleotide sequence analysis of the $95 \mathrm{~kb} \mathrm{3}$ terminal region of the murine T-cell receptor $\alpha / \delta$ chain locus: strategy and methodology. Genomics 13: 1198-1208, 1992

Winoto, A. and Baltimore, D. A novel, inducible and T cell specific enhancer located at the $3^{\prime}$ end of the T cell receptor $\alpha$ locus. $E M B O$ $J$ 8: 729-733, 1989

Ylug, I. G., See, C. G., and Fisher, E. M. C. The DAD1 protein, whose defect causes apoptotic cell death, maps to human chromosome 14 . Genomics 26: 433-435, 1995 